\title{
Erratum to: "Structural Analysis of Aluminum Oxyhydroxide Aerogel: Small Angle Scattering Studies" [Journal of Surface Investigation: $X$-ray, Synchrotron and Neutron Techniques 12, 296 (2018)]
}

A. N. Khodan ${ }^{a, ~ *}$, G. P. Kopitsa ${ }^{b, c}$, Kh. E. Yorov ${ }^{d}$, A. E. Baranchikov ${ }^{e}$, V. K. Ivanov ${ }^{e, f}$, A. Feoktystov ${ }^{g}$, and V. Pipich ${ }^{g}$

${ }^{a}$ Frumkin Institute of Physical Chemistry and Electrochemistry, Russian Academy of Sciences, Moscow, 119071 Russia

${ }^{b}$ Konstantinov Petersburg Nuclear Physics Institute, National Research Center "Kurchatov Institute”, Gatchina, 188300 Russia

${ }^{c}$ Grebenshchikov Institute of Silicate Chemistry, Russian Academy of Sciences, St.-Petersburg, 199034 Russia

${ }^{d}$ Moscow State University, Moscow, 119991 Russia

${ }^{e}$ Kurnakov Institute of General and Inorganic Chemistry, Russian Academy of Sciences, Moscow, 119071 Russia

${ }^{f}$ National Research Tomsk State University, Tomsk, 634050 Russia

${ }^{g}$ Julich Centre for Neutron Science, Forschungszentrum Julich GmbH, Outstation at MLZ, Garching, Germany

*e-mail: anatole.khodan@gmail.com

Submitted April 26, 2018

DOI: $10.1134 / \mathrm{S} 1027451018030394$

The title of the article should read as follows:

Structural Analysis of Aluminum Oxyhydroxide Aerogel: Small Angle Scattering Studies 